\title{
Erratum to: Studies on Immunogenicity and Antigenicity of Baculovirus-Expressed Binding Region of Plasmodium falciparum EBA-140 Merozoite Ligand
}

\author{
Agata Zerka ${ }^{1}$ - Joanna Rydzak ${ }^{1}$ - Anna Lass ${ }^{2}$ - Beata Szostakowska ${ }^{2}$. \\ Wacław Nahorski ${ }^{3}$ - Agnieszka Wroczyńska ${ }^{3}$ - Przemyslaw Myjak ${ }^{2}$. \\ Hubert Krotkiewski ${ }^{1}$ Ewa Jaskiewicz ${ }^{1,4}$
}

Published online: 19 November 2015

(C) L. Hirszfeld Institute of Immunology and Experimental Therapy, Wroclaw, Poland 2015

\section{Erratum to: Arch Immunol Ther Exp DOI 10.1007/s00005-015-0367-5}

The author would like to amend the Acknowledgment section of his online published article. The complete Acknowledgment section should read as follows:

The research was supported by Grants No. N N302 281436 from the Ministry of Science and Higher Education of Poland and Grant No. 2012/05/N/NZ6/00667 from the National Centre of Science. The publication was supported by Wroclaw Centre of Biotechnology, programme The Leading National Research Centre (KNOW) for the years 2014-2018.

The online version of the original article can be found under doi:10.1007/s00005-015-0367-5.

Ewa Jaskiewicz

ewa.jaskiewicz@iitd.pan.wroc.pl

1 Ludwik Hirszfeld Institute of Immunology and Experimental Therapy, Polish Academy of Sciences, Wrocław, Poland

2 Department of Tropical Parasitology, Institute of Maritime and Tropical Medicine in Gdynia, Medical University of Gdańsk, Gdańsk, Poland

3 Department of Tropical and Parasitic Diseases, Institute of Maritime and Tropical Medicine in Gdynia, Medical University of Gdańsk, Gdańsk, Poland

4 Department of Molecular Biology, Faculty of Biological Sciences, University of Zielona Góra, Zielona Gora, Poland 